\title{
On efficient estimation of population mean under non-response
}

\author{
Shashi Bhushan ${ }^{a}$, Abhay Pratap Pandey ${ }^{1, b}$ \\ ${ }^{a}$ Department of Mathematics and Statistics, D. S. M. N. R. University, Lucknow, India; \\ ${ }^{b}$ Ramanujan College, University of Delhi, India
}

\begin{abstract}
The present paper utilizes auxiliary information to neutralize the effect of non-response for estimating the population mean. Improved ratio type estimators for population mean have been proposed and their properties are studied. These estimators are suggested for both single phase sampling and two phase sampling in presence of non-response. Empirical studies are conducted to validate the theoretical results and demonstrate the performance of the proposed estimators. The proposed estimators are shown to perform better than those used by Cochran (Sampling Techniques (3rd ed), John Wiley \& Sons, 1977), Khare and Srivastava (In ProceedingsNational Academy Science, India, Section A, 65, 195-203, 1995), Rao (Randomization Approach in Incomplete Data in Sample Surveys, Academic Press, 1983; Survey Methodology 12, 217-230, 1986), and Singh and Kumar (Australian \& New Zealand Journal of Statistics, 50, 395-408, 2008; Statistical Papers, 51, 559-582, 2010) under the derived optimality condition. Suitable recommendations are put forward for survey practitioners.
\end{abstract}

Keywords: auxiliary variable, non-response, ratio type estimator, mean square error, efficiency

\section{Introduction}

The problem of non-response is inevitable in most sample surveys because the information cannot be obtained from all units selected in the survey due to various reasons. An estimator based on such incomplete information is biased and the final outcome may be misleading, when the respondents differ from non-respondents. In their seminal paper Hansen and Hurwitz (1946) considered a technique of sub-sampling the non-respondents in order to adjust for the non-response bias in a mail survey.

In sampling theory, it is well known that the efficiency of the estimators of unknown population parameters of the study variable can be increased by suitably using known information on an auxiliary variable. The ratio, product and regression methods of estimation are good examples in this context. Non-response adversely affects the estimate of population mean and population variance; in addition, many authors have suggested a number of estimators to estimate population parameter and their variance under the non-response for various situations. Cochran (1977) and Rao (1986) suggested a ratio method to estimate the population mean $\bar{Y}$ of the study variate $y$ with sub-sampling from non-respondents.

Khare and Srivastava (1995) suggested an estimation procedure of the population mean using an auxiliary character in the presence of non-response, Khare and Srivastava (1995) proposed the studying of a conventional and alternative two phase sampling ratio, product and regression estimators in

\footnotetext{
${ }^{1}$ Corresponding author: Abhay Pratap Pandey, Ramanujan College, University of Delhi, Kalkaji New Delhi-110019, India. E-mail: abhaypratap.pandey@gmail.com
}

Published 31 January 2019 / journal homepage: http://csam.or.kr

(c) 2019 The Korean Statistical Society, and Korean International Statistical Society. All rights reserved. 
the presence of non-response. Khare and Srivastava (1997) proposed transformed ratio type estimators for the population mean in the presence of non-response. Okafor and Lee (2000) proposed a double sampling scheme for ratio and regression estimation with sub sampling; in addition, the nonrespondent also deal with the non-response problem. Khare and Srivastava (1993). Singh and Kumar (2008) proposed a general class of population mean estimators in survey sampling using auxiliary information with sub sampling for the non-respondent. Singh et al. (2010) also suggested a number of estimators to estimate population mean under non-response. Khare and Kumar (2011) proposed a method to estimate the population mean using known coefficient of variation of the study character in the presence of non-response. Singh and Bhushan (2012) proposed a generalized classes of two phase sampling estimators of population mean in presence of non-response. Shabbir and Khan (2013) also suggested a number of estimators to estimate the finite population mean using two auxiliary variables in two phase sampling in the presence of non-response. Sunil Kumar (Kumar, 2015) suggested an efficient use of auxiliary information in estimating the population ratio, product and mean in the presence of non-response.

An interesting finding of all these papers was that the regression (difference) estimators were found to be best in terms of mean squared error (MSE); in addition, any ratio type estimator can at best attain the MSE of these regression (difference) estimators. In this paper, we have proposed some improvement over regression as well as ratio estimators proposed by various authors in their earlier works.

\section{Notations and existing results}

Hansen and Hurwitz (1946) considered mail surveys in the first attempt-, and personal interviews in the second attempt. In the Hansen \& Hurwitz method, the population of size of $N$ is supposed to be composed of two strata, namely respondents and non-respondents; having sizes $N_{1}$ and $N_{2}\left(=N-N_{1}\right)$. Thus we label the data as $y_{1}, \ldots, y_{N_{1}}$ for the response group, and as $y_{N_{1}+1}, \ldots, y_{N_{1}+N_{2}}$ for the nonresponse group. Let $\bar{Y}=\sum_{i=1}^{N} y_{i} / N$ and $S_{y}^{2}=\sum_{i=1}^{N}\left(y_{i}-\bar{Y}\right)^{2} /(N-1)$ denote the population mean and variance, respectively. Let $\bar{Y}_{1}=\sum_{i=1}^{N_{1}} y_{i} / N_{1}$ and $S_{y_{1}}^{2}=\sum_{i=1}^{N_{1}}\left(y_{i}-\bar{Y}_{1}\right)^{2} /\left(N_{1}-1\right)$ denote the mean and variance of the response group, respectively. Similarly, let $\bar{Y}_{2}=\sum_{i=N_{1}}^{N_{1}+N_{2}} y_{i} / N_{2}$ and $S_{y_{2}}^{2}=\sum_{i=N_{1}+1}^{N_{1}+N_{2}}\left(y_{i}-\right.$ $\left.\bar{Y}_{2}\right)^{2} /\left(N_{2}-1\right)$ denote the mean and variance of the non-response group, respectively. The population mean can be written as $\bar{Y}=W_{1} \bar{Y}_{1}+W_{2} \bar{Y}_{2}, W_{1}=N_{1} / N$, and $W_{2}=N_{2} / N$. Let us consider a random sample of size $n$ are drawn by using simple random sampling without replacement (SRSWOR). The random sample $n$ should be made of two strata, namely $n_{1}$ respondents and $\left(n-n_{1}\right)$ non-respondents. The sample mean $\bar{y}_{1}=\sum_{i=1}^{n_{1}} y_{i} / n_{1}$ is unbiased for $\bar{Y}_{1}$, but has a bias equal to $W_{1}\left(\bar{Y}_{1}-\bar{Y}_{2}\right)$ in estimating the population mean $\bar{Y}$. The sample mean $\bar{y}_{2 r}=\sum_{i=1}^{r} y_{i} / r$ is unbiased for the mean $\bar{y}_{2}$ of the $n_{2}$ units. An unbiased estimator for the population mean $\bar{Y}$ is

$$
\bar{y}^{*}=w_{1} \bar{y}_{1}+w_{2} \bar{y}_{2},
$$

where $w_{1}=n_{1} / n$ and $w_{2}=n_{2} / n$. The variance of $\bar{y}^{*}$ is given by

$$
\operatorname{Var}\left(\bar{y}^{*}\right)=P S_{y}^{2}+Q S_{y_{2}}^{2}
$$

where $P=(1-f) / n, Q=W_{2}(k-1) / n$, and $f=n / N$.

Let $x_{i}(i=1,2, \ldots, N)$ denote an auxiliary variate correlated with study variate $y_{i}(i=1,2, \ldots, N)$. The population mean of the auxiliary variable $x$ is $\bar{X}=\sum_{i=1}^{N} x_{i} / N$. Let $\bar{X}_{1}$ and $\bar{X}_{2}$ denote the means of the response and non-response groups. Let $\bar{x}$ denote the mean of all the $n$ units. Let $\bar{x}_{1}$ and $\bar{x}_{2}$ denote 
the means of the $n_{1}$ responding units and the $n_{2}$ non-responding units. Further let $\bar{x}_{2 r}=\sum_{i=1}^{r} x_{i} / r$ denote the mean of the subsampled units. The population variances of $x$ and $y$ are denoted by $S_{x}^{2}$ and $S_{y}^{2}$, and the population covariance by $S_{x y}$. The population correlation coefficient is $\rho=S_{x y} / S_{x}^{2} S_{y}^{2}$. The unbiased estimator of the population mean $\bar{X}$ of the auxiliary variable $x$ is

$$
\bar{x}^{*}=w_{1} \bar{x}_{1}+w_{2} \bar{x}_{2} .
$$

The variance of $\bar{x}^{*}$ is given by

$$
\operatorname{Var}\left(\bar{x}^{*}\right)=P S_{x}^{2}+Q S_{x_{2}}^{2}
$$

where $S_{x_{2}}^{2}=\sum_{i=N_{1}+1}^{N_{1}+N_{2}}\left(x_{i}-\bar{X}_{2}\right)^{2} /\left(N_{2}-1\right)$.

Similarly, In two phase sampling, we have $n^{\prime}$ observations on $x$ from the first-phase sample, $n_{1}$ observations on $y$ from the responding units of the $n$ second-phase sample units, and $r$ observations on $y$ from the subsample units selected from the $n_{2}$ non response units of the second-phase sample. Let $\bar{x}^{\prime}$ be the sample mean of auxiliary variable $x$ based on the first-phase sample. Using the information on the auxiliary variable $x$ collected from the first-phase sample, Cochran (1977), Khare and Srivastava (1995), Rao (1986), Okafor and Lee (2000), and Singh and Kumar (2008, 2010) suggested ratio and regression type estimators under non-response. We have classified these estimators into seven different strategies depending upon the available auxiliary information under both single phase sampling and two phase sampling.

Strategy I: When $\bar{y}^{*}, \bar{x}^{*}$, and $\bar{X}$ are used. If the auxiliary variable is known, the non-response occurs on the study variable $y$ and information on the auxiliary variable $x$ is not available from all the sample units along the population mean $\bar{X}$. The ratio and regression type estimators are

$$
\begin{aligned}
t_{r_{1}} & =\bar{y}^{*}\left(\frac{\bar{X}}{\bar{x}^{*}}\right), \\
t_{R_{1}} & =\bar{y}^{*}\left(\frac{\bar{X}}{\bar{x}^{*}}\right),{ }_{1} \\
t_{1} & =\bar{y}^{*}+b^{*}\left(\bar{X}-\bar{x}^{*}\right),
\end{aligned}
$$

where $b^{*}=s_{x y}^{*} / s_{x}^{*^{2}}$ and the estimates $s_{x y}^{*}$ and $s_{x}^{*^{2}}$ are based on the available data. To the first order of approximation, the MSEs of estimators $t_{r_{1}}, t_{R_{1}}$, and $t_{1}$ are given by

$$
\begin{aligned}
\operatorname{MSE}\left(t_{r_{1}}\right) & =P\left(S_{y}^{2}+R^{2} S_{x}^{2}-2 R S_{x y}\right)+Q\left(S_{y_{2}}^{2}+R^{2} S_{x_{2}}^{2}-2 R S_{x y_{2}}\right), \\
\min \cdot \operatorname{MSE}\left(t_{R_{1}}\right) & =P S_{y}^{2}+Q S_{y_{2}}^{2}-\left\{\frac{\left(P S_{x y}+Q S_{x y_{2}}\right)^{2}}{P S_{y}^{2}+Q S_{y_{2}}^{2}}\right\}, \\
\min \cdot \operatorname{MSE}\left(t_{1}\right) & =P S_{y}^{2}\left(1-\rho^{2}\right)+Q\left(S_{y_{2}}^{2}+\beta^{2} S_{x_{2}}^{2}-2 \beta S_{x y_{2}}\right),
\end{aligned}
$$

where $R=\bar{Y} / \bar{X}, \beta=S_{x y} / S_{x}^{2}$, and $\beta_{1 \mathrm{opt}}=\left(P S_{x y}+Q S_{x y_{2}}\right) /\left(P S_{y}^{2}+Q S_{y_{2}}^{2}\right)$.

Strategy II: When $\bar{y}^{*}, \bar{x}$, and $\bar{X}$ are used. The non-response occurs on the study variable $y$, and information on the auxiliary variable $x$ is available from all the sample units along the population 
mean $\bar{X}$ of the auxiliary variable is known. The ratio and regression type estimators are

$$
\begin{aligned}
t_{r_{2}} & =\bar{y}^{*}\left(\frac{\bar{X}}{\bar{x}}\right), \\
t_{R_{2}} & =\bar{y}^{*}\left(\frac{\bar{X}}{\bar{x}}\right)^{\beta_{2}}, \\
t_{2} & =\bar{y}^{*}+b(\bar{X}-\bar{x}),
\end{aligned}
$$

where $b=s_{x y}^{*} / s_{x}^{2}$. The first order approximate MSEs of the estimators $t_{r_{2}}, t_{R_{2}}$, and $t_{2}$ are given by

$$
\begin{aligned}
\operatorname{MSE}\left(t_{r_{3}}\right) & =P\left(S_{y}^{2}+R^{2} S_{x}^{2}-2 R S_{x y}\right)+Q S_{y_{2}}^{2}, \\
\min \cdot \operatorname{MSE}\left(t_{R_{2}}\right) & =\min \cdot \operatorname{MSE}\left(t_{2}\right)=P S_{y}^{2}\left(1-\rho^{2}\right)+Q S_{y_{2}}^{2},
\end{aligned}
$$

where $\beta_{2}=S_{x y_{2}} / S_{x_{2}}^{2}$ and $\beta_{2 \text { opt }}=S_{x y} / S_{x}^{2}$.

Strategy III: When $\bar{y}^{*}, \bar{x}^{*}$, and $\bar{x}$ are used. The non-response occurs on the study variable $y$, and the information on the auxiliary variable $x$ is obtained from all the sample units, but the population mean $\bar{X}$ of the auxiliary variable is not known. The ratio and regression type estimators are

$$
\begin{aligned}
t_{r_{3}} & =\bar{y}^{*}\left(\frac{\bar{x}}{\bar{x}^{*}}\right), \\
t_{R_{3}} & =\bar{y}^{*}\left(\frac{\bar{x}}{\bar{x}^{*}}\right)^{\beta_{3}}, \\
t_{3} & =\bar{y}^{*}+b_{(2 r)}\left(\bar{x}-\bar{x}^{*}\right),
\end{aligned}
$$

where $b_{(2 r)}=s_{x y(2 r)} / s_{x_{(2 r)}}^{2}$. The MSEs to the first order of approximation, of the estimators $t_{r_{3}}, t_{R_{3}}$, and $t_{3}$ are given by

$$
\begin{aligned}
\operatorname{MSE}\left(t_{r_{3}}\right) & =P S_{y}^{2}+Q\left(S_{y_{2}}^{2}+R^{2} S_{x_{2}}^{2}-2 R S_{x y_{2}}\right), \\
\min \cdot \operatorname{MSE}\left(t_{R_{3}}\right) & =\min \cdot \operatorname{MSE}\left(t_{3}\right)=P S_{y}^{2}+Q S_{y_{2}}^{2}\left(1-\rho_{2}^{2}\right),
\end{aligned}
$$

where $\beta_{3 \mathrm{opt}}=S_{x y_{2}} / S_{x_{2}}^{2}$.

Strategy IV: When $\bar{y}^{*}, \bar{x}, \bar{x}^{*}$, and $\bar{X}$ are used. Singh and Kumar (2008) suggested the estimators given below

$$
\begin{aligned}
t_{r_{4}} & =\bar{y}^{*}\left(\frac{\bar{X}}{\bar{x}^{*}}\right)\left(\frac{\bar{X}}{\bar{x}}\right), \\
t_{R_{4}} & =\bar{y}^{*}\left(\frac{\bar{X}}{\bar{x}^{*}}\right)^{\beta_{4}}\left(\frac{\bar{X}}{\bar{x}}\right)^{\beta_{5}}, \\
t_{4} & =\bar{y}^{*}+d_{1}\left(\bar{x}-\bar{x}^{*}\right)+d_{2}(\bar{X}-\bar{x}) .
\end{aligned}
$$

To the first order of approximation, the MSEs of the estimators $t_{r_{4}}, t_{R_{4}}$, and $t_{4}$ are given by

$$
\begin{aligned}
\operatorname{MSE}\left(t_{r_{4}}\right) & =P\left\{S_{y}^{2}+4 R S_{x}^{2}(R-\beta)\right\}+Q\left\{S_{y_{2}}^{2}+R S_{x_{2}}^{2}\left(R-2 \beta_{(2)}\right)\right\}, \\
\min \cdot \operatorname{MSE}\left(t_{4}\right) & =\min \cdot \operatorname{MSE}\left(t_{R_{4}}\right)=P S_{y}^{2}\left(1-\rho^{2}\right)+Q S_{y_{2}}^{2}\left(1-\rho_{2}^{2}\right),
\end{aligned}
$$


where optimum values of $\beta_{4}, \beta_{5}, d_{1}$, and $d_{2}$ are given by $\beta_{4 \mathrm{opt}}=\beta_{(2)} / R, \beta_{5 \mathrm{opt}}=\beta-\beta_{(2)} / R, d_{1 \mathrm{opt}}=\beta_{(2)}$, and $d_{2 \text { opt }}=\beta$. Now, we shall consider three more strategies under the two-phase sampling scheme proposed by Okafor and Lee (2000) and Singh and Kumar (2010).

Strategy V: When $\bar{y}^{*}, \bar{x}^{*}$, and $\bar{x}^{\prime}$ are used. Okafor and Lee (2000) proposed a double sampling scheme for ratio estimation for sub sampling the non-respondent that also deals with the non-response problem.

$$
\begin{aligned}
t_{r_{5}} & =\bar{y}^{*}\left(\frac{\bar{x}^{\prime}}{\bar{x}^{*}}\right) \\
t_{R_{5}} & =\bar{y}^{*}\left(\frac{\bar{x}^{\prime}}{\bar{x}^{*}}\right)^{\beta_{6}} \\
t_{5} & =\bar{y}^{*}+b^{*}\left(\bar{x}^{\prime}-\bar{x}^{*}\right)
\end{aligned}
$$

To the first order of approximation, the MSEs of the estimators $t_{r_{5}}, t_{R_{5}}$, and $t_{5}$ are given by

$$
\begin{aligned}
\operatorname{MSE}\left(t_{r_{5}}\right) & =T S_{y}^{2}+S\left(S_{y}^{2}+R^{2} S_{x}^{2}-2 R S_{x y}\right)+Q\left(S_{y_{2}}^{2}+R^{2} S_{x_{2}}^{2}-2 R S_{x y_{2}}\right), \\
\min \cdot \operatorname{MSE}\left(t_{R_{5}}\right) & =\min \cdot \operatorname{MSE}\left(t_{5}\right)=T S_{y}^{2}+S S_{y}^{2}\left(1-\rho^{2}\right)+Q\left\{S_{y_{2}}^{2}+\beta S_{x_{2}}^{2}\left(\beta-2 \beta_{(2)}\right)\right\},
\end{aligned}
$$

where $\beta_{6 o p t}=\left\{(P-T) S_{x y}+Q S_{x y_{2}}\right\} /\left(P S_{x}^{2}+Q S_{x_{2}}^{2}\right), S=\left(1 / n-1 / n^{\prime}\right)$, and $T=\left(1 / n^{\prime}-1 / N\right)$.

Strategy VI: When $\bar{y}^{*}, \bar{x}$, and $\bar{x}^{\prime}$ are used. Khare and Srivastava (1995) and Okafor and Lee (2000) proposed a double sampling scheme for ratio estimation with sub sampling the non-respondent that also deals with the non-response problem.

$$
\begin{aligned}
t_{r_{6}} & =\bar{y}^{*}\left(\frac{\bar{x}^{\prime}}{\bar{x}}\right), \\
t_{R_{6}} & =\bar{y}^{*}\left(\frac{\bar{x}^{\prime}}{\bar{x}}\right)^{\beta_{7}}, \\
t_{6} & =\bar{y}^{*}+b^{* *}\left(\bar{x}^{\prime}-\bar{x}\right) .
\end{aligned}
$$

To the first order of approximation, the MSE's of the estimators $t_{r_{6}}, t_{R_{6}}$, and $t_{6}$ are given by

$$
\begin{aligned}
\operatorname{MSE}\left(t_{r_{6}}\right) & =T S_{y}^{2}+S\left(S_{y}^{2}+R^{2} S_{x}^{2}-2 R S_{x y}\right)+Q S_{y_{2}}^{2}, \\
\min \cdot \operatorname{MSE}\left(t_{R_{6}}\right) & =\min \cdot \operatorname{MSE}\left(t_{6}\right)=T S_{y}^{2}+S S_{y}^{2}\left(1-\rho^{2}\right)+Q S_{y_{2}}^{2},
\end{aligned}
$$

where $\beta_{7 \mathrm{opt}}=S_{x y} / S_{x}^{2}$.

Strategy VII: When $\bar{y}^{*}, \bar{x}^{*}, \bar{x}$, and $\bar{x}^{\prime}$ are used. Singh and Kumar (2008) gave estimators for the population mean $\bar{Y}$ by using a double sampling scheme under non-response, which are mentioned as:

$$
\begin{aligned}
t_{r_{7}} & =\bar{y}^{*}\left(\frac{\bar{x}^{\prime}}{\bar{x}^{*}}\right)\left(\frac{\bar{x}^{\prime}}{\bar{x}}\right), \\
t_{R_{7}} & =\bar{y}^{*}\left(\frac{\bar{x}^{\prime}}{\bar{x}^{*}}\right)^{\beta_{8}}\left(\frac{\bar{x}^{\prime}}{\bar{x}}\right)^{\beta_{9}}, \\
t_{7} & =\bar{y}^{*}+d_{3}\left(\bar{x}-\bar{x}^{*}\right)+d_{4}\left(\bar{x}^{\prime}-\bar{x}\right) .
\end{aligned}
$$


To the first order of approximation, the MSEs of the above estimators are given by

$$
\begin{aligned}
\operatorname{MSE}\left(t_{r_{7}}\right) & =S\left\{S_{y}^{2}+4 R S_{x}^{2}(R-\beta)\right\}+Q\left\{S_{y_{2}}^{2}+R S_{x_{2}}^{2}\left(R-2 \beta_{(2)}\right)\right\}+T S_{y}^{2}, \\
\min \cdot \operatorname{MSE}\left(t_{R_{7}}\right) & =\min \cdot \operatorname{MSE}\left(t_{7}\right)=T S_{y}^{2}+S S_{y}^{2}\left(1-\rho^{2}\right)+Q S_{y_{2}}^{2}\left(1-\rho_{2}^{2}\right),
\end{aligned}
$$

where optimum values of $\beta_{8}, \beta_{9}, d_{3}$, and $d_{4}$ are given by $\beta_{8 \mathrm{opt}}=\beta_{(2)} / R, \beta_{9 \mathrm{opt}}=\beta / R, d_{3 \mathrm{opt}}=\beta_{(2)}$, and $d_{\text {topt }}=\beta$.

Bhushan and Pandey (2017) proposed some improved regression type estimators under nonresponse in seven different strategies using Searls methodology (Searls, 1964). These estimators were an improvement over the corresponding regression estimators, which are BLUE, under non-response in seven different strategies stated as follow.

$$
\begin{aligned}
& T_{1}=\gamma_{1} \bar{y}^{*}+\delta_{1}\left(\bar{X}-\bar{x}^{*}\right), \\
& T_{2}=\gamma_{2} \bar{y}^{*}+\delta_{2}(\bar{x}-\bar{x}), \\
& T_{3}=\gamma_{3} \bar{y}^{*}+\delta_{3}\left(\bar{x}-\bar{x}^{*}\right), \\
& T_{4}=\gamma_{4} \bar{y}^{*}+\delta_{4}\left(\bar{x}-\bar{x}^{*}\right)+\eta_{1}(\bar{X}-\bar{x}), \\
& T_{5}=\gamma_{5} \bar{y}^{*}+\delta_{5}\left(\bar{x}^{\prime}-\bar{x}^{*}\right), \\
& T_{6}=\gamma_{6} \bar{y}^{*}+\delta_{6}\left(\bar{x}^{\prime}-\bar{x}\right), \\
& T_{7}=\gamma_{7} \bar{y}^{*}+\delta_{7}\left(\bar{x}-\bar{x}^{*}\right)+\eta_{2}\left(\bar{x}^{\prime}-\bar{x}\right),
\end{aligned}
$$

where $\gamma_{i}, \delta_{j}$, and $\eta_{k}\{i=j=1,2, \ldots, 7 ; k=1,2\}$ are suitably (optimally) chosen scalars to optimize MSE. The MSE of the estimators are given by

$$
\min \cdot \operatorname{MSE}\left(T_{i}\right)=\frac{\bar{Y}^{2} \operatorname{MSE}\left(t_{i}\right)}{\bar{Y}^{2}+\operatorname{MSE}\left(t_{i}\right)}, \quad i=1,2, \ldots, 7
$$

obviously,

$$
\min \cdot \operatorname{MSE}\left(T_{i}\right)<\operatorname{MSE}\left(t_{i}\right) .
$$

The optimal values of $\gamma_{i}, \delta_{j}$, and $\eta_{k}\{i=j=1,2, \ldots, 7 ; k=1,2\}$ are given by

$$
\begin{aligned}
& \gamma_{1}=\frac{\bar{Y}^{2}}{\left\{\bar{Y}^{2}+P S_{y}^{2}+Q S_{y_{2}}^{2}-\frac{\left(P S_{x y}+Q S_{x y_{2}}\right)^{2}}{\left(Q S_{x}^{2}+Q S_{x_{2}}^{2}\right)}\right\}}, \quad \gamma_{2}=\frac{\bar{Y}^{2}}{\left\{\bar{Y}^{2}+P S_{y}^{2}\left(1-\rho^{2}\right)+Q S_{y_{2}}^{2}\right\}}, \\
& \gamma_{3}=\frac{\bar{Y}^{2}}{\left\{\bar{Y}^{2}+P S_{y}^{2}+Q S_{y_{2}}^{2}\left(1-\rho_{2}^{2}\right)\right\}}, \quad \gamma_{4}=\frac{\bar{Y}^{2}}{\left\{\bar{Y}^{2}+P S_{y}^{2}\left(1-\rho^{2}\right)+Q S_{y_{2}}^{2}\left(1-\rho_{2}^{2}\right)\right\}}, \\
& \gamma_{5}=\frac{\bar{Y}^{2}}{\left\{\bar{Y}^{2}+P S_{y}^{2}+Q S_{y_{2}}^{2}-\frac{\left(S S_{x y}+Q S_{x y_{2}}\right)^{2}}{\left(S S_{x}^{2}+Q S_{x_{2}}^{2}\right)}\right\}}, \quad \gamma_{6}=\frac{\bar{Y}^{2}}{\left\{\bar{Y}^{2}+T S_{y}^{2}+S S_{y}^{2}\left(1-\rho^{2}\right)+Q S_{y_{2}}^{2}\right\}}, \\
& \gamma_{7}=\frac{\bar{Y}^{2}}{\left\{\bar{Y}^{2}+T S_{y}^{2}+S S_{y}^{2}\left(1-\rho^{2}\right)+Q S_{y_{2}}^{2}\left(1-\rho_{2}^{2}\right)\right\}},
\end{aligned}
$$




$$
\begin{aligned}
& \delta_{1}=\frac{\bar{Y}^{2}\left(P S_{x y}+Q S_{x y_{2}}\right)}{\left(P S_{x}^{2}+Q S_{x_{2}}^{2}\right)\left\{\bar{Y}^{2}+P S_{y}^{2}+Q S_{y_{2}}^{2}-\frac{\left(P S_{x y}+Q S_{x_{2}}\right)^{2}}{\left(P S_{x}^{2} Q Q S_{x_{2}}\right)}\right\}}, \quad \delta_{2}=\frac{\bar{Y}^{2} S_{x y}}{S_{x}^{2}\left\{\bar{Y}^{2}+P S_{y}^{2}\left(1-\rho^{2}\right)+Q S_{y_{2}}^{2}\right\}}, \\
& \delta_{3}=\frac{\bar{Y}^{2} S_{x y_{2}}}{S_{x_{2}}^{2}\left\{\bar{Y}^{2}+P S_{y}^{2}+Q S_{y_{2}}^{2}\left(1-\rho_{2}^{2}\right)\right\}}, \quad \delta_{4}=\frac{\bar{Y}_{x y_{2}}}{S_{x_{2}}^{2}\left\{\bar{Y}^{2}+P S_{y}^{2}\left(1-\rho^{2}\right)+Q S_{y_{2}}^{2}\left(1-\rho_{2}^{2}\right)\right\}}, \\
& \delta_{5}=\frac{\bar{Y}^{2}\left(S S_{x y}+Q S_{x y_{2}}\right)}{\left(S S_{x}^{2}+Q S_{x_{2}}^{2}\right)\left\{\bar{Y}^{2}+P S_{y}^{2}+Q S_{y_{2}}^{2}-\frac{\left(S S_{x y}+B S_{x_{2}}\right)^{2}}{\left(S S_{x}^{2}+Q S_{x_{2}}^{2}\right)}\right\}}, \quad \delta_{6}=\frac{\bar{Y}_{x y}^{2} S_{x y}}{S_{x}^{2}\left\{\bar{Y}^{2}+T S_{y}^{2}+S S_{y}^{2}\left(1-\rho^{2}\right)+Q S_{y_{2}}^{2}\right\}}, \\
& \delta_{7}=\frac{\bar{Y}^{2} S_{x y_{2}}}{S_{x_{2}}^{2}\left\{\bar{Y}^{2}+T S_{y}^{2}+S S_{y}^{2}\left(1-\rho^{2}\right)+Q S_{y_{2}}^{2}\left(1-\rho_{2}^{2}\right)\right\}}, \\
& \eta_{1}=\frac{\bar{Y}^{2} S_{x y}}{S_{x}^{2}\left\{\bar{Y}^{2}+P S_{y}^{2}\left(1-\rho^{2}\right)+Q S_{y_{2}}^{2}\left(1-\rho_{2}^{2}\right)\right\}}, \quad \eta_{2}=\frac{\bar{Y}^{2} S_{x y}}{S_{x}^{2}\left\{\bar{Y}^{2}+T S_{y}^{2}+S S_{y}^{2}\left(1-\rho^{2}\right)+Q S_{y_{2}}^{2}\left(1-\rho_{2}^{2}\right)\right\}},
\end{aligned}
$$

where $P=(1 / n-1 / N), Q=W_{2}(k-1) / n, S=\left(1 / n-1 / n^{\prime}\right)$, and $T=\left(1 / n^{\prime}-1 / N\right)$.

Bhushan and Pandey (2017) showed that the these estimators were better than conventional regression estimators. In this article, we propose some new ratio type estimators and compared these with the corresponding regression estimators given earlier. The proposed estimators are motivated by Cochran (1977), Khare and Srivastava (1995), Rao (1986), Okafor and Lee (2000), and Singh and Kumar (2008) under the one phase and two phase sampling using seven different strategies under non-response.

\section{Proposed improved ratio type estimators under a non-response}

We propose improved ratio type estimators using Searls methodology (Searls, 1964), Searls (1964) proposed a technique to improve the conventional estimators by multiplying a tuning constant term $\alpha$ whose optimum value depends on the coefficient of variation, which is a fairly stable quantity, and we refer this technique of multiplication by a tuning constant $\alpha$ as Searls type transformation (STT), under seven different strategies in single phase sampling and two phase sampling, as follows.

$$
T_{s}=\alpha \bar{y}^{*}
$$

optimum value of $\alpha$ is given by

$$
\alpha=\frac{\bar{Y}^{2}}{\bar{Y}^{2}+P S_{y}^{2}+Q S_{y_{2}}^{2}} .
$$

The proposed estimator under Strategy I, when $\bar{y}^{*}, \bar{x}^{*}$, and $\bar{X}$ are used, is given by

$$
T_{s_{1}}=\alpha_{1} \bar{y}^{*}\left(\frac{\bar{X}}{\bar{x}^{*}}\right)^{\beta_{1}} .
$$

The proposed estimator under Strategy II, when $\bar{y}^{*}, \bar{x}$, and $\bar{X}$ are used, is given by

$$
T_{s_{2}}=\alpha_{2} \bar{y}^{*}\left(\frac{\bar{X}}{\bar{x}}\right)^{\beta_{2}} \text {. }
$$


The proposed estimator under Strategy III, when $\bar{y}^{*}, \bar{x}^{*}$, and $\bar{x}$ are used, is given by

$$
T_{s_{3}}=\alpha_{3} \bar{y}^{*}\left(\frac{\bar{x}}{\bar{x}^{*}}\right)^{\beta_{3}}
$$

The proposed estimator under Strategy IV, when $\bar{y}^{*}, \bar{x}, \bar{x}^{*}$, and $\bar{X}$ are used, is given by

$$
T_{s_{4}}=\alpha_{4} \bar{y}^{*}\left(\frac{\bar{X}}{\bar{x}^{*}}\right)^{\beta_{4}}\left(\frac{\bar{X}}{\bar{x}}\right)^{\beta_{5}} .
$$

The proposed estimator under Strategy $\mathrm{V}$, when $\bar{y}^{*}, \bar{x}^{*}$, and $\bar{x}^{\prime}$ are used, is given by

$$
T_{s_{5}}=\alpha_{5} \bar{y}^{*}\left(\frac{\bar{x}^{\prime}}{\bar{x}^{*}}\right)^{\beta_{6}} \text {. }
$$

The proposed estimator under Strategy VI, when $\bar{y}^{*}, \bar{x}$, and $\bar{x}^{\prime}$ are used, is given by

$$
T_{s_{6}}=\alpha_{6} \bar{y}^{*}\left(\frac{\bar{x}^{\prime}}{\bar{x}}\right)^{\beta_{7}} \text {. }
$$

The proposed estimator under Strategy VII, when $\bar{y}^{*}, \bar{x}^{*}, \bar{x}$, and $\bar{x}^{\prime}$ are used, is given by

$$
T_{s_{7}}=\alpha_{7} \bar{y}^{*}\left(\frac{\bar{x}^{\prime}}{\bar{x}^{*}}\right)^{\beta_{8}}\left(\frac{\bar{x}^{\prime}}{\bar{x}}\right)^{\beta_{9}},
$$

where $\alpha_{j}(j=1,2, \ldots 7)$ is a suitable chosen scalars. And the optimum values of $\alpha_{j}$ are defined in appendix.

Theorem 1. The bias and minimum MSE of the new ratio type estimator $T_{s_{j}}(j=1,2, \ldots 7)$ is given by

$$
\operatorname{Bias}\left(T_{s_{j}}\right)=\bar{Y}\left(\alpha_{j}-1\right)
$$

and

$$
\min . M S E_{\alpha_{j}}\left(T_{s_{j}}\right)=\bar{Y}^{2}\left(1-\frac{B_{j}^{2}}{A_{j}}\right)
$$

$j=1,2, \ldots, 7$.

Proof: See Appendix.

It is interesting to note that simultaneous optimization with respect to the characterizing scalars $\gamma_{i}$ and $\delta_{i}$ of the expression (2.5) of MSE is possible for regression (difference) type estimators. But simultaneous optimization with respect to the characterizing scalars $\alpha_{i}$ and $\beta_{i}$ of the expression (3.2) not possible for ratio type estimators.

Theorem 2. The proposed ratio type estimators $T_{s_{j}},(j=1,2, \ldots, 7)$ are better than difference type estimators $T_{k},(k=1,2, \ldots, 7)$ iff

$$
\frac{B_{j}^{2}}{A_{j}}>\gamma_{k_{o p t}}
$$


and vice versa. Otherwise both are equally efficient in case of equality in (3.3).

Proof: It may be easily observed from (2.5) that the MSE of the difference type estimators $T_{i},(i=$ $1,2, \ldots, 7)$ are given by

$$
\min . M S E\left(T_{i}\right)=\bar{Y}^{2}\left(1-\gamma_{i_{o p t}}\right)
$$

Comparing (3.4) with (3.2), we have the theorem.

The only way ascertain (3.3) if this holds in practice is through a computational study.

Theorem 3. The proposed ratio type estimators $T_{s_{j}}, j=1,2, \ldots, 7$ are better than the conventional ratio type estimators $t_{R_{j}}, j=1,2, \ldots, 7$ iff

$$
\frac{B_{j}^{2}}{A_{j}}>\left\{1-\frac{\min \cdot M S E\left(t_{R_{j}}\right)}{\bar{Y}^{2}}\right\}
$$

and vice versa. Otherwise both are equally efficient in case of equality in (3.5).

Proof: It may be easily observed from (3.2) and (2.2), we have the theorem.

Theorem 4. The proposed ratio type estimators $T_{s_{j}}, j=1,2, \ldots, 7$ are better than the conventional regression type estimators $t_{i}, i=1,2, \ldots, 7$ iff

$$
\frac{B_{j}^{2}}{A_{j}}>\left\{1-\frac{\min \cdot M \operatorname{SSE}\left(t_{i}\right)}{\bar{Y}^{2}}\right\}
$$

and vice versa. Otherwise both are equally efficient in case of equality in (3.6).

Proof: It may be easily observed from (3.2) and (2.4), we have the theorem.

\section{Empirical study}

In order to have a better understanding about the efficiency of the proposed estimators we have conducted a comprehensive empirical study on three populations and compared the proposed estimators with the existing estimators. The percentage relative efficiency (PRE) is calculated as

$$
\operatorname{PRE}=\frac{\operatorname{Var}\left(\bar{y}^{*}\right)}{\min \cdot \operatorname{MSE}\left(T_{s_{j}}\right)} \times 100, \quad j=1,2, \ldots, 7 .
$$

1. The first population considered by Srivastava $(1993, p .50)$ consists of a list of 70 villages in the administrative devision of Tehsil India that includes population and cultivated area (in acres) data from 1981. Here the cultivated area (in acres) is taken as the main study character and the population of village is taken as the auxiliary character.The parameters of the population are as follows: $N=70, n^{\prime}=40, n=25, \bar{Y}=981.29, \bar{X}=1755.53, S_{y}=613.66, S_{x}=1406.13, \bar{Y}_{2}=597.29$, $\bar{X}_{2}=1100.24, S_{y_{2}}=244.11, S_{x_{2}}=631.51, \rho=0.778, \rho_{2}=0.445, R=0.5589, \beta=0.3395$, $\beta_{2}=0.1720, W_{2}=0.20$.

2. The second population considered by Khare and Kumar (2011). For the population of 96 villages of rural areas under Police Station, Singur, District Hooghly from district census Handbook (1981), 
Table 1: Mean squared error and percentage relative efficiency of the estimators with respect to $\bar{y}^{*}$ for population 1

\begin{tabular}{cccccc}
\hline \hline \multirow{2}{*}{ Estimator } & & \multicolumn{3}{c}{$k$} \\
\cline { 2 - 6 } & & 1 & 2 & 3 & 4 \\
\hline \multirow{3}{*}{ Strategy I } & $\bar{y}^{*}$ & $10160.17(100.00)$ & $10636.88(100.00)$ & $11113.60(100.00)$ & $11590.32(100.00)$ \\
& $T_{s}$ & $10054.08(101.05)$ & $10520.67(101.10)$ & $10986.79(101.15)$ & $11452.47(101.20)$ \\
\hline \multirow{3}{*}{ Strategy II } & $t_{R_{1}}$ & $4288.77(236.90)$ & $4745.94(224.13)$ & $5195.20(213.92)$ & $5637.74(205.58)$ \\
& $T_{1}$ & $4269.75(237.95)$ & $4722.66(225.23)$ & $5167.32(215.07)$ & $5604.92(206.78)$ \\
& $T_{s_{1}}$ & $4247.51(\mathbf{2 3 9 . 2 0})$ & $4696.51(\mathbf{2 2 6 . 4 8})$ & $5137.05(\mathbf{2 1 6 . 3 4})$ & $5570.35(\mathbf{2 0 8 . 0 7})$ \\
\hline \multirow{3}{*}{ Strategy III } & $t_{R_{2}}$ & $4298.93(236.34)$ & $4775.65(222.73)$ & $5252.36(211.59)$ & $5729.08(202.31)$ \\
& $T_{2}$ & $4279.82(237.39)$ & $4752.08(223.83)$ & $5223.87(212.74)$ & $5695.19(203.51)$ \\
& $T_{s_{2}}$ & $4259.43(\mathbf{2 3 8 . 5 3})$ & $4729.86(\mathbf{2 2 4 . 8 9})$ & $5199.82(\mathbf{2 1 3 . 7 3})$ & $5669.32(\mathbf{2 0 4 . 4 4})$ \\
\hline \multirow{3}{*}{ Strategy IV } & $t_{R_{3}}$ & $10065.76(100.94)$ & $10448.08(101.81)$ & $10830.39(102.61)$ & $11212.71(103.37)$ \\
& $T_{3}$ & $9961.63(101.99)$ & $10335.93(102.91)$ & $10709.93(103.77)$ & $11083.65(104.57)$ \\
& $T_{s_{3}}$ & $9959.45(\mathbf{1 0 2 . 0 1})$ & $10331.38(\mathbf{1 0 2 . 9 6})$ & $10702.83(\mathbf{1 0 3 . 8 4})$ & $11073.81(\mathbf{1 0 4 . 6 6})$ \\
\hline \multirow{3}{*}{ Strategy V } & $t_{R_{4}}$ & $4204.53(241.65)$ & $4586.84(231.90)$ & $4969.16(223.65)$ & $5351.47(216.58)$ \\
& $T_{4}$ & $4186.25(242.70)$ & $4565.09(233.00)$ & $4961.16(224.80)$ & $5321.89(217.78)$ \\
& $T_{s_{4}}$ & $4164.88(\mathbf{2 4 3 . 9 5})$ & $4540.74(\mathbf{2 3 4 . 2 5})$ & $4916.11(\mathbf{2 2 6 . 0 6})$ & $5291.00(\mathbf{2 1 9 . 0 6})$ \\
\hline \multirow{3}{*}{ Strategy VI } & $t_{R_{5}}$ & $6727.54(151.02)$ & $7182.84(148.09)$ & $7638.14(145.50)$ & $8093.44(143.21)$ \\
& $T_{5}$ & $6680.87(152.07)$ & $7123.29(149.32)$ & $7554.66(147.11)$ & $7977.40(145.29)$ \\
& $T_{s_{5}}$ & $6662.06(\mathbf{1 5 2 . 5 1})$ & $7104.86(\mathbf{1 4 9 . 7 1})$ & $7546.58(\mathbf{1 4 7 . 2 6})$ & $7987.23(\mathbf{1 4 5 . 1 1})$ \\
\hline \multirow{3}{*}{ Strategy VII } & $t_{R_{6}}$ & $6741.11(150.72)$ & $7217.83(147.37)$ & $7694.55(144.43)$ & $8171.26(141.84)$ \\
& $T_{6}$ & $6694.24(151.77)$ & $7164.13(148.47)$ & $7633.55(145.58)$ & $8102.50(143.04)$ \\
& $T_{s_{6}}$ & $6677.77(\mathbf{1 5 2 . 1 5})$ & $7146.59(\mathbf{1 4 8 . 8 4})$ & $7614.95(\mathbf{1 4 5 . 9 4})$ & $8082.86(\mathbf{1 4 3 . 3 9})$ \\
\hline \hline & $t_{R_{7}}$ & $6646.71(152.86)$ & $7029.02(151.33)$ & $7411.34(149.95)$ & $7793.66(148.71)$ \\
& $T_{7}$ & $6601.14(153.91)$ & $6978.08(152.43)$ & $7354.73(151.10)$ & $7731.08(149.91)$ \\
& $T_{s_{7}}$ & $6583.19(\mathbf{1 5 4 . 3 3 )}$ & $6957.40(\mathbf{1 5 2 . 8 8})$ & $7331.14(\mathbf{1 5 1 . 5 9})$ & $7704.39(\mathbf{1 5 0 . 4 4 )}$ \\
\hline
\end{tabular}

published by the government of India, the data on the number of cultivators $y$, as a study character and the population of villages, as an auxiliary character $x$ have been taken. The non-response rate in the population is considered to be $25 \%$. The values of the parameters of the population are given as follows: $N=96, n^{\prime}=65, n=25, \bar{Y}=185.22, \bar{X}=1807.23, S_{y}=195.03, S_{x}=1921.77$, $S_{y_{2}}=97.82, S_{x_{2}}=1068.44, \rho=0.904, \rho_{2}=0.895, R=0.1025, W_{2}=0.25$.

3. Third population considered from Srivastava (1993, p.50). The data belongs to the data on physical growth of upper-socio-economic group of 95 school children of Varanasi under an ICMR study. The first $25 \%$ (i.e., 24 children) units have been considered as non-response units. The values of the parameters related to the study variate $y$ (weight in $\mathrm{kg}$ ) and the auxiliary variate $x$ (chest circumference in cm) have been given below: $N=95, n^{\prime}=70, n=35, \bar{Y}=19.497, \bar{X}=55.8611$, $S_{y}=3.0435, S_{x}=3.2735, S_{y_{2}}=2.3552, S_{x_{2}}=2.5137, \rho=0.8460, \rho_{2}=0.7290, R=0.3490$, $\beta=0.7865, \beta_{2}=0.6829, W_{2}=0.25, N_{2}=24, N_{1}=71$.

From perusal of above results it is observed that the new ratio type estimators proposed $T_{s_{i}}$ are always better than the conventional ratio type counterparts $t_{R_{i}}$. Hence, we conclude that all proposed new ratio type estimators have higher efficiency in comparison to the conventional ratio type estimators. A comparison of STD (or regression) estimators $T_{i}(i=1,2, \ldots, 7)$ with new ratio type estimators $T_{s_{i}}(i=1,2, \ldots, 7)$, we observe that the new ratio type estimators are always better than the estimators proposed by Bhushan and Pandey regression type estimators under optimality condition (3.3). Therefore, in population I and II proposed ratio type estimators $T_{s_{j}}$ are better than Bhushan and Pandey regression type estimators $T_{i}$, as (3.3) is satisfied. However, the case is reversed for population III. 
Table 2: Mean squared error and percentage relative efficiency of the estimators with respect to $\bar{y}^{*}$ for population 2

\begin{tabular}{|c|c|c|c|c|c|}
\hline \multirow{2}{*}{\multicolumn{2}{|c|}{ Estimator }} & \multicolumn{4}{|c|}{$k$} \\
\hline & & 1 & 2 & 3 & 4 \\
\hline & $\bar{y}^{*}$ & $1220.94(100.00)$ & $1316.63(100.00)$ & $1412.31(100.00)$ & $1508.00(100.00)$ \\
\hline & $T_{s}$ & $1178.98(103.56)$ & $1267.96(103.84)$ & $1356.47(104.12)$ & $1444.51(104.40)$ \\
\hline \multirow{3}{*}{ Strategy I } & $t_{R_{1}}$ & $225.71(540.93)$ & $245.57(536.15)$ & $265.30(532.34)$ & $284.93(529.25)$ \\
\hline & $T_{1}$ & $224.23(544.49)$ & 243.83 (539.99) & $263.26(536.46)$ & $282.58(533.65)$ \\
\hline & $T_{s_{1}}$ & $223.29(\mathbf{5 4 6 . 8 0})$ & $242.63(\mathbf{5 4 2 . 6 5})$ & $261.79(\mathbf{5 3 9 . 4 8})$ & $280.80(\mathbf{5 3 7 . 0 3})$ \\
\hline \multirow{3}{*}{ Strategy II } & $t_{R_{2}}$ & $301.36(405.14)$ & $397.05(331.61)$ & $492.74(286.62)$ & $588.43(256.28)$ \\
\hline & $T_{2}$ & 298.74 (408.69) & 392.51(335.44) & $485.76(290.74)$ & $578.50(260.67)$ \\
\hline & $T_{s_{2}}$ & 297.73 (410.08) & 391.21 (336.55) & 484.18 (291.69) & $576.64(\mathbf{2 6 1 . 5 1})$ \\
\hline \multirow{3}{*}{ Strategy III } & $t_{R_{3}}$ & $1144.29(106.70)$ & $1163.33(113.18)$ & $1182.37(119.45)$ & $1201.41(125.52)$ \\
\hline & $T_{3}$ & $1107.36(110.26)$ & $1125.18(117.01)$ & $1142.98(123.56)$ & $1160.76(129.91)$ \\
\hline & $T_{s_{3}}$ & 1106.75 (110.32) & $1123.95(\mathbf{1 1 7 . 1 4})$ & $1141.10(\mathbf{1 2 3 . 7 7})$ & $1158.21(\mathbf{1 3 0 . 2 0})$ \\
\hline \multirow{3}{*}{ Strategy IV } & $t_{R_{4}}$ & $224.72(312.90)$ & $243.76(291.08)$ & $262.79(277.11)$ & $281.84(267.40)$ \\
\hline & $T_{4}$ & $223.25(546.88)$ & $242.04(543.98)$ & $260.80(541.53)$ & $279.54(539.46)$ \\
\hline & $T_{s_{4}}$ & 222.32 (549.18) & $240.86(\mathbf{5 4 6 . 6 3})$ & $259.36(\mathbf{5 4 4 . 5 4})$ & $277.81(\mathbf{5 4 2 . 8 2})$ \\
\hline \multirow{3}{*}{ Strategy V } & $t_{R_{5}}$ & $380.12(226.28)$ & $400.02(223.52)$ & $419.93(221.57)$ & $439.83(220.11)$ \\
\hline & $T_{5}$ & 375.95 (324.76) & $395.33(333.05)$ & 414.54 (340.69) & 433.63 (347.76) \\
\hline & $T_{s_{5}}$ & 374.68 (325.86) & $393.68(\mathbf{3 3 4 . 4 4})$ & 412.61 (342.28) & 431.48 (349.49) \\
\hline \multirow{3}{*}{ Strategy VI } & $t_{R_{6}}$ & $455.79(184.51)$ & $551.48(162.44)$ & $647.16(149.51)$ & $742.85(141.01)$ \\
\hline & $T_{6}$ & $449.81(271.43)$ & $542.75(242.58)$ & $635.18(222.35)$ & $727.11(207.40)$ \\
\hline & $T_{s_{6}}$ & $448.60(\mathbf{2 7 2 . 1 7})$ & $541.30(\mathbf{2 4 3 . 2 3})$ & $633.50(\mathbf{2 2 2 . 9 4})$ & 725.19 (207.94) \\
\hline \multirow{3}{*}{ Strategy VII } & $t_{R_{7}}$ & $379.14(227.20)$ & $398.18(224.87)$ & $417.22(223.21)$ & $436.26(221.97)$ \\
\hline & $T_{7}$ & 374.99 (325.59) & $393.61(334.50)$ & $412.21(342.62)$ & $430.78(350.06)$ \\
\hline & $T_{s_{7}}$ & 373.74 (326.68) & 392.05 (335.83) & 410.31 (344.20) & 428.53 (351.90) \\
\hline
\end{tabular}

\section{Simulation study}

In this section, simulation is conducted to evaluate the performance of the proposed class of estimators with respect to traditional estimators. For this study we have generated a population size $N=1,000$ from standard normal distribution using MVRNORM package in software R, where study and auxiliary variable are correlated with correlation $\rho=0.7$, draw sample of size $n=200$ with 35\% non-response. The whole simulation process starting from the drawing sample from variable $Y$ and auxiliary variable $X$ from normal population and calculating the estimates was repeated 50,000 times.

\section{Conclusions}

From the above computational results as shown in Tables 1-3, it may be concluded that the proposed estimators $T_{s_{j}}$ dominate the over conventional ratio type estimators $t_{R_{i}}$. The most interesting and noticeable finding of this paper is the vitiation of conventional thought, that the ratio type estimator can at most match upto its regression estimator. Consequently in such a manner, we proved that the proposed ratio type estimators $T_{s_{j}}(j=1,2, \ldots, 7)$ provides an improvement over traditional ratio type estimators counterpart $t_{R_{i}}(i=1,2, \ldots, 7)$ while satisfying (3.5); in addition, we also proved that the proposed ratio estimator can provide an improvement over both traditional regression estimators $t_{i}(i=$ $1,2, \ldots, 7)$ and proposed regression estimators $T_{i}(i=1,2, \ldots, 7)$ under the optimality conditions (3.3). Thus the proposed estimators are highly rewarding in terms of the increased precession of the estimates and negative impact of the non-response. Therefore, the proposed estimators may be recommended to survey practitioners for real-life applications. 
Table 3: Mean squared error and percentage relative efficiency of the estimators with respect to $\bar{y}^{*}$ for population 3

\begin{tabular}{|c|c|c|c|c|c|}
\hline \multirow{2}{*}{\multicolumn{2}{|c|}{ Estimator }} & \multicolumn{4}{|c|}{$k$} \\
\hline & & 1 & 2 & 3 & 4 \\
\hline & $\bar{y}^{*}$ & $0.2067(100.000)$ & $0.2464(100.000)$ & $0.2860(100.000)$ & $0.3256(100.000)$ \\
\hline & $T_{s}$ & $0.2066(100.054)$ & $0.2462(100.064)$ & $0.2857(100.075)$ & $0.3253(100.085)$ \\
\hline \multirow{3}{*}{ Strategy I } & $t_{R_{1}}$ & $0.0664(311.050)$ & $0.0853(288.830)$ & $0.1040(274.840)$ & $0.1227(265.220)$ \\
\hline & $T_{1}$ & 0.0664 (311.104) & 0.0852 (288.893) & $0.1040(\mathbf{2 7 4 . 9 1 3})$ & $0.1227(\mathbf{2 6 5 . 3 0 7})$ \\
\hline & $T_{s_{1}}$ & $0.0664(311.060)$ & $0.0853(288.840)$ & $0.1040(274.860)$ & $0.1227(265.250)$ \\
\hline \multirow{3}{*}{ Strategy II } & $t_{R_{2}}$ & $0.0871(237.290)$ & $0.1267(194.380)$ & $0.1663(171.900)$ & $0.2060(158.070)$ \\
\hline & $T_{2}$ & $0.0871(\mathbf{2 3 7 . 3 4 2})$ & 0.1267 (194.441) & 0.1663 (171.977) & $0.2058(\mathbf{1 5 8 . 1 5 8})$ \\
\hline & $T_{s_{2}}$ & $0.0871(237.310)$ & $0.1267(194.410)$ & $0.1663(171.950)$ & $0.2059(158.130)$ \\
\hline \multirow{3}{*}{ Strategy III } & $t_{R_{3}}$ & $0.1857(111.340)$ & $0.2043(120.610)$ & $0.2228(128.350)$ & $0.2414(134.890)$ \\
\hline & $T_{3}$ & 0.1856 (111.392) & 0.2041 (120.680) & $0.2227(\mathbf{1 2 8 . 4 2 1})$ & $0.2412(\mathbf{1 3 4 . 9 7 4})$ \\
\hline & $T_{s_{3}}$ & $0.1856(111.390)$ & $0.2042(120.670)$ & $0.2227(128.410)$ & $0.2412(134.960)$ \\
\hline \multirow{3}{*}{ Strategy IV } & $t_{R_{4}}$ & $0.0660(312.890)$ & $0.0846(291.080)$ & $0.1032(277.110)$ & $0.1217(267.400)$ \\
\hline & $T_{4}$ & $0.0660(\mathbf{3 1 2 . 9 5 0})$ & $0.0846(\mathbf{2 9 1 . 1 4 4 )}$ & $0.1031(\mathbf{2 7 7 . 1 8 6})$ & $0.1217(\mathbf{2 6 7 . 4 8 6})$ \\
\hline & $T_{s_{4}}$ & $0.0660(312.910)$ & $0.0846(291.090)$ & $0.1032(277.130)$ & $0.1217(267.430)$ \\
\hline \multirow{3}{*}{ Strategy V } & $t_{R_{5}}$ & $0.0913(226.280)$ & $0.1102(223.520)$ & $0.1290(221.570)$ & $0.1479(220.110)$ \\
\hline & $T_{5}$ & 0.0913 (226.331) & 0.1101 (223.692) & 0.1288 (221.952) & $0.1475(\mathbf{2 2 0 . 7 2 2})$ \\
\hline & $T_{s_{5}}$ & $0.0913(226.300)$ & $0.1102(223.550)$ & $0.1290(221.600)$ & $0.1479(220.150)$ \\
\hline \multirow{3}{*}{ Strategy VI } & $t_{R_{6}}$ & $0.1120(184.510)$ & $0.1516(162.440)$ & $0.1913(149.510)$ & $0.2309(141.010)$ \\
\hline & $T_{6}$ & $0.1120(184.568)$ & $0.1516(162.503)$ & 0.1912 (149.581) & $0.2307(\mathbf{1 4 1 . 0 9 8})$ \\
\hline & $T_{s_{6}}$ & $0.1120184 .540)$ & $0.1516(162.480)$ & $0.1912(149.560)$ & $0.2308(141.080)$ \\
\hline \multirow{3}{*}{ Strategy VII } & $t_{R_{7}}$ & $0.0910(227.200)$ & 0.1095 (224.870) & $0.1281(223.210)$ & 0.1467 (221.970) \\
\hline & $T_{7}$ & 0.0909 (227.259) & 0.1095 (224.934) & $0.1280(\mathbf{2 2 3 . 2 8 6})$ & $0.1466(\mathbf{2 2 2 . 0 5 7})$ \\
\hline & $T_{s_{7}}$ & $0.0909(227.230)$ & $0.1095(224.890)$ & $0.1281(223.240)$ & $0.1467(222.010)$ \\
\hline
\end{tabular}

Table 4: Percentage relative efficiency (PRE) of the proposed estimators with respect to $\bar{y}^{*}$ using simulation

\begin{tabular}{|c|c|c|}
\hline \multicolumn{2}{|c|}{ Estimator } & \multirow{2}{*}{$\begin{array}{c}\text { PRE } \\
100\end{array}$} \\
\hline & $\bar{y}^{*}$ & \\
\hline & $T_{s}$ & 100.086 \\
\hline \multirow{3}{*}{ Strategy I } & $t_{R_{1}}$ & 165.163 \\
\hline & $T_{1}$ & 166.775 \\
\hline & $T_{s_{1}}$ & 167.016 \\
\hline \multirow{3}{*}{ Strategy II } & $t_{R_{2}}$ & 142.838 \\
\hline & $T_{2}$ & 143.0174 \\
\hline & $T_{s_{2}}$ & 143.637 \\
\hline \multirow{3}{*}{ Strategy III } & $t_{R_{3}}$ & 120.771 \\
\hline & $T_{3}$ & 121.753 \\
\hline & $T_{s_{3}}$ & 120.795 \\
\hline \multirow{3}{*}{ Strategy IV } & $t_{R_{4}}$ & 168.449 \\
\hline & $T_{4}$ & 168.503 \\
\hline & $T_{s_{4}}$ & 168.727 \\
\hline \multirow{3}{*}{ Strategy V } & $t_{R_{5}}$ & 148.608 \\
\hline & $T_{5}$ & 149.331 \\
\hline & $T_{s_{5}}$ & 149.486 \\
\hline \multirow{3}{*}{ Strategy VI } & $t_{R_{6}}$ & 130.484 \\
\hline & $T_{6}$ & 130.495 \\
\hline & $T_{s_{6}}$ & 131.025 \\
\hline \multirow{3}{*}{ Strategy VII } & $t_{R_{7}}$ & 153.491 \\
\hline & $\begin{array}{l}T_{7} \\
T_{7}\end{array}$ & 153.893 \\
\hline & $T_{s_{7}}$ & 154.491 \\
\hline
\end{tabular}




\section{Appendix: Outline of derivation of Theorem 1}

The MSE of $T_{s_{j}}(j=1,2, \ldots, 7)$ is given by The MSE of $T_{s_{1}}$ is given by

$$
\begin{aligned}
\operatorname{MSE}\left(T_{s_{1}}\right)= & \bar{Y}^{2}\left[1+\alpha_{1}^{2}\left\{1+P\left(C_{y}^{2}+\beta_{1} C_{x}^{2}\left(2 \beta_{1}+1\right)-4 \beta_{1} \rho C_{y} C_{x}\right)+Q\left(C_{y_{2}}^{2}+\beta_{1} C_{x_{2}}^{2}\left(2 \beta_{1}+1\right)-4 \beta_{1} \rho C_{y_{2}} C_{x_{2}}\right)\right\}\right. \\
& \left.-2 \alpha_{1}\left\{1+P\left(\frac{\beta_{1}}{2}\left(\beta_{1}+1\right) C_{x}^{2}-\beta_{1} \rho C_{y} C_{x}\right)+Q\left(\frac{\beta_{1}}{2}\left(\beta_{1}+1\right) C_{x_{2}}^{2}-2 \beta_{1} \rho C_{y_{2}} C_{x_{2}}\right)\right\}\right]
\end{aligned}
$$

which can be expressed as

$$
\operatorname{MSE}\left(T_{s_{1}}\right)=\bar{Y}^{2}\left[1+\alpha_{1}^{2} A_{1}-2 \alpha_{1} B_{1}\right]
$$

For optimum value of $\alpha_{1}$ differentiating the $\operatorname{MSE}\left(T_{s_{1}}\right)$ with respect to $\alpha_{1}$ and equating to zero we get,

$$
\alpha_{1 \mathrm{opt}}=\frac{B_{1}}{A_{1}}
$$

substituting the optimum value of $\alpha_{1}$ in $\operatorname{MSE}\left(T_{s_{1}}\right)$ we get minimum MSE

$$
\min \cdot \operatorname{MSE}\left(T_{s_{1}}\right)=\bar{Y}^{2}\left(1-\frac{B_{1}^{2}}{A_{1}}\right)
$$

The derivations for other estimators $T_{s_{j}}(j=1,2, \ldots, 7)$ can be done on similar lines. We then have

$$
\operatorname{MSE}\left(T_{s_{j}}\right)=\bar{Y}^{2}\left[1+\alpha_{j}^{2} A_{j}-2 \alpha_{j} B_{j}\right] .
$$

It is important to mention here that simultaneous optimization w.r.t, $\alpha_{j}$ and $\beta_{j}$ of the expression of MSE is not possible and we use optimum value of $\beta_{j}=\beta_{\text {jopt }}$ when $\alpha_{j}=1$ and use this within $\alpha_{j}=\alpha_{\text {jopt }}$ to obtain (3.2) as used recently by various authors including Singh and Solanki (2013).

$$
\min . \mathrm{MSE}_{\alpha_{j}}\left(T_{s_{j}}\right)=\bar{Y}^{2}\left(1-\frac{B_{j}^{2}}{A_{j}}\right)
$$

The optimum values of scalars for different estimators involved are given as

$$
\begin{aligned}
A_{1}= & \bar{Y}^{2}+P\left\{S_{y}^{2}+\beta_{1} R^{2} S_{x}^{2}\left(2 \beta_{1}+1\right)-4 \beta_{1} R S_{x y}\right\}+Q\left\{S_{y_{2}}^{2}+\beta_{1} R^{2} S_{x_{2}}^{2}\left(2 \beta_{1}+1\right)-4 \beta_{1} R S_{x y_{2}}\right\} \\
B_{1}= & \bar{Y}^{2}+P\left\{\frac{\beta_{1}}{2} R^{2} S_{x}^{2}\left(\beta_{1}+1\right)-\beta_{1} R S_{x y}\right\}+Q\left\{\frac{\beta_{1}}{2} R^{2} S_{x_{2}}^{2}\left(\beta_{1}+1\right)-2 \beta_{1} R S_{x y_{2}}\right\} \\
A_{2}= & \bar{Y}^{2}+P\left\{S_{y}^{2}+\beta_{2} R^{2} S_{x}^{2}\left(2 \beta_{2}+1\right)-4 \beta_{2} R S_{x y}\right\}+Q S_{y_{2}}^{2}, \\
B_{2}= & \bar{Y}^{2}+P\left\{\frac{\beta_{2}}{2} R^{2} S_{x}^{2}\left(\beta_{2}+1\right)-\beta_{2} R S_{x y}\right\} \\
A_{3}= & \bar{Y}^{2}+P S_{y}^{2}+Q\left\{S_{y_{2}}^{2}+\beta_{3} R^{2} S_{x_{2}}^{2}\left(2 \beta_{3}+1\right)-4 \beta_{3} R S_{x y_{2}}\right\}, \\
B_{3}= & \bar{Y}^{2}+Q\left\{\frac{\beta_{3}}{2} R^{2} S_{x_{2}}^{2}\left(\beta_{3}+1\right)-\beta_{3} R S_{x y_{2}}\right\}, \\
A_{4}= & \bar{Y}^{2}+P\left\{S_{y}^{2}+\beta_{4} R^{2} S_{x}^{2}\left(2 \beta_{4}+1\right)+\beta_{5} R^{2} S_{x}^{2}\left(2 \beta_{5}+1\right)-4 \beta_{4} R S_{x y}-4 \beta_{5} R S_{x y}+4 \beta_{4} \beta_{5} R^{2} S_{x}^{2}\right\} \\
& +Q\left\{S_{y_{2}}^{2}+\beta_{4} R^{2} S_{x_{2}}^{2}\left(2 \beta_{4}+1\right)-4 \beta_{4} R S_{x y_{2}}\right\},
\end{aligned}
$$




$$
\begin{aligned}
B_{4}= & \bar{Y}^{2}+P\left\{\frac{\beta_{4}}{2} R^{2} S_{x}^{2}\left(\beta_{4}+1\right)+\frac{\beta_{5}}{2} R^{2} S_{x}^{2}\left(\beta_{5}+1\right)-\beta_{4} R S_{x y}-\beta_{5} R S_{x y}+\beta_{4} \beta_{5} R^{2} S_{x}^{2}\right\} \\
& +Q\left\{\frac{\beta_{4}}{2} R^{2} S_{x_{2}}^{2}\left(\beta_{4}+1\right)-\beta_{4} R S_{x y_{2}}\right\}, \\
A_{5}= & \bar{Y}^{2}+P\left(S_{y}^{2}+\beta_{6} R^{2} S_{x}^{2}\left(2 \beta_{6}+1\right)-4 \beta_{6} R S_{x y}\right)+Q\left(S_{y_{2}}^{2}+\beta_{6} R^{2} S_{x_{2}}^{2}\left(2 \beta_{6}+1\right)-4 \beta_{6} R S_{x y_{2}}\right) \\
& -T\left(\beta_{6} R^{2} S_{x}^{2}\left(2 \beta_{6}+1\right)-4 \beta_{6} R S_{x y}\right), \\
B_{5}= & \bar{Y}^{2}+P\left\{\frac{\beta_{6}}{2} R^{2} S_{x}^{2}\left(\beta_{6}+1\right)-\beta_{6} R S_{x y}\right\}+Q\left\{\frac{\beta_{6}}{2} R^{2} S_{x_{2}}^{2}\left(\beta_{6}+1\right)-\beta_{6} R S_{x y_{2}}\right\} \\
& -T\left\{\frac{\beta_{6}}{2} R^{2} S_{x}^{2}\left(\beta_{6}+1\right)-\beta_{6} R S_{x y}\right\}, \\
A_{6}= & \bar{Y}^{2}+P\left(S_{y}^{2}+\beta_{7} R^{2} S_{x}^{2}\left(2 \beta_{7}+1\right)-4 \beta_{7} R S_{x y}\right)+Q S_{y_{2}}^{2}-T\left(\beta_{7} R^{2} S_{x}^{2}\left(2 \beta_{7}+1\right)-4 \beta_{7} R S_{x y}\right), \\
B_{6}= & \bar{Y}^{2}+P\left\{\frac{\beta_{7}}{2} R^{2} S_{x}^{2}\left(\beta_{7}+1\right)-\beta_{7} R S_{x y}\right\}-T\left\{\frac{\beta_{7}}{2} R^{2} S_{x}^{2}\left(\beta_{7}+1\right)-\beta_{7} R S_{x y}\right\}, \\
A_{7}= & \bar{Y}^{2}+P\left(S_{y}^{2}+\beta_{9} R^{2} S_{x}^{2}\left(2 \beta_{9}+1\right)-4 \beta_{9} R S_{x y}\right)+Q\left(S_{y_{2}}^{2}+\beta_{8} R^{2} S_{x_{2}}^{2}\left(2 \beta_{8}+1\right)-4 \beta_{8} R S_{x y_{2}}\right) \\
& -T\left(\beta_{9} R^{2} S_{x}^{2}\left(2 \beta_{9}+1\right)-4 \beta_{9} R S_{x y}\right), \\
B_{7}= & \bar{Y}^{2}+P\left\{\frac{\beta_{9}}{2} R^{2} S_{x}^{2}\left(\beta_{9}+1\right)-\beta_{9} R S_{x y}\right\}+Q\left\{\frac{\beta_{8}}{2} R^{2} S_{x_{2}}^{2}\left(\beta_{8}+1\right)-\beta_{8} R S_{x y_{2}}\right\} \\
& -T\left\{\frac{\beta_{9}}{2} R^{2} S_{x}^{2}\left(\beta_{9}+1\right)-\beta_{9} R S_{x y}\right\},
\end{aligned}
$$

where $P=(1 / n-1 / N), Q=W_{2}(k-1) / n, S=\left(1 / n-1 / n^{\prime}\right)$, and $T=\left(1 / n^{\prime}-1 / N\right)$.

\section{References}

Bhushan S and Pandey AP (2017). An efficient estimation procedure for population mean under non-response, (Submitted).

Cochran WG (1977). Sampling Techniques (3rd ed), John Wiley \& Sons, New York.

Hansen MH and Hurwitz WN (1946). The problem of non-response in sample surveys, Journal of the American Statistical Association, 41, 517-529.

Khare BB and Kumar S (2011). Estimation of population mean using known coefficient of variation of the study character in the presence of non-response, Communications in Statistics - Theory and Methods, 40, 2044-2058.

Khare BB and Srivastava S (1993). Estimation of population mean using auxiliary character in presence of non-response, National Academy Science Letters-India, 16, 111-114.

Khare BB and Srivastava S (1995). Study of conventional and alternative two-phase sampling ratio, product and regression estimators in presence of non-response. In Proceedings-National Academy Science, India, Section A, 65, 195-203.

Khare BB and Srivastava S (1997). Transformed ratio type estimators for the population mean in the presence of non-response, Communications in Statistics - Theory and Methods, 26, 1779-1791.

Kumar S (2015). Efficient use of auxiliary information in estimating the population ratio, product and mean in presence of non-response, Journal of Advanced Computing, 4, 68-87.

Okafor FC and Lee H (2000). Double sampling for ratio and regression estimation with sub sampling the non-respondents, Survey Methodology, 26, 183-188.

Rao PSRS (1983). Randomization Approach in Incomplete Data in Sample Surveys, Vol. 2, WG 
Madow, I Olkin, and DB Rubin (Eds), pp. 33-44, Academic Press, New York.

Rao PSRS (1986). Ratio estimation with subsampling the nonrespondents, Survey Methodology 12, 217-230.

Searls DT (1964). The Utilization of a Known Coefficient of Variation in the Estimation Procedure, Journal of the American Statistical Association, 59, 1225-1226.

Shabbir J and Khan NS (2013). On estimating the finite population mean using two auxiliary variables in two phase sampling in the presence of non response, Communications in Statistics - Theory and Methods, 42, 4127-4145.

Singh HP and Kumar S (2008). A regression approach to the estimation of the finite population mean in the presence of non-response, Australian \& New Zealand Journal of Statistics, 50, 395-408.

Singh HP and Kumar S (2010). Estimation of mean in presence of non-response using two phase sampling scheme, Statistical Papers, 51, 559-582.

Singh HP, Kumar S, and Kozak M (2010). Improved estimation of finite population mean using subsampling to deal with non response in two-phase sampling scheme, Communications in Statistics - Theory and Methods, 39, 791-802.

Singh HP and Solanki RS (2013). An efficient class of estimators for the population mean using auxiliary information, Communications in Statistics - Theory and Methods, 42, 145-163.

Singh RK and Bhushan S (2012). Generalized classes of two phase sampling estimators of population mean in presence of non-response. In Proceeding of VII ISOS, Aligarh Muslim University, Aligarh.

Srivastava S (1993). Some problems on the estimation of population mean using auxiliary character in presence of non-response in sample surveys (Ph.D thesis), BHU, Varanasi, U.P., India. 
